\title{
DETERMINAÇÃO EXPERIMENTAL DO CALOR ESPECÍFICO DO SUCO DE LARANJA
}

\author{
L.. V. D. OLIVEIRA ${ }^{1} ;$ N. R. OLIVEIRA ${ }^{1}$ e M. B. SILVA ${ }^{2}$ \\ ${ }^{1}$ Universidade Federal do Triângulo Mineiro, Departamento de Engenharia Química \\ ${ }^{2}$ Universidade Federal do Triângulo Mineiro, Departamento de Engenharia Química \\ E-mail para contato: bacciuftm@gmail.com
}

\begin{abstract}
RESUMO - Dados referentes às propriedades térmicas de sucos de frutas são ainda escassos na literatura. Sendo assim, o presente trabalho teve como objetivo determinar experimentalmente o calor específico do suco de laranja pelo método proposto por Hwang e Hayakawa, através da modificação de uma garrafa térmica como calorímetro. Os resultados obtidos foram coerentes quando comparados aos da literatura, comprovando a eficácia deste método.
\end{abstract}

\section{INTRODUÇÃO}

O aumento da demanda por alimentos no país e no mundo faz com que haja aumento também na necessidade de modernização e um maior desenvolvimento tecnológico, com o objetivo de produzir mais, além de melhorar e conservar de maneira mais duradoura os produtos.

A indústria de sucos tropicais no país necessita dimensionar os equipamentos utilizados no processamento, principalmente aqueles destinados à transferência de calor e isto exigem dados precisos de propriedades térmicas dos produtos, tais como condutividade térmica, difusividade térmica e calor específico.

Polpas de frutas estão sujeitas a processos de resfriamento e aquecimento. Quando o alimento não é consumido imediatamente, existe a necessidade de ele ser processado, incluindo ser resfriado ou congelado para conservação de suas características.

Na literatura já existem trabalhos publicados sobre propriedades térmicas. Polley et al (1980), por exemplo, publicaram tabelas dessas propriedades para diversos alimentos, entre eles carnes, frutas, legumes, cereais e também diversos sucos, porém estão incompletas.

Valores experimentais de difusividade térmica de sucos de frutas tropicais, tais como maça, cereja, uva, laranja e tomate foram publicados no trabalho de George (1990). Resultados significativos na determinação das propriedades termofísicas de diversos sucos foram apresentados por Telis-Romero et al (1998).

Apesar da existência de trabalhos científicos já publicados, vale ressaltar que o Brasil é o maior produtor e exportador de laranja, detendo $50 \%$ de toda produção mundial, sendo que apenas $3 \%$ é 
destinada ao mercado interno. Há um crescente aumento da demanda por produtos alimentícios no país. Visando atender as exigências de modernização, aumentando a qualidade técnica dos processos industriais, maiores informações sobre propriedades físicas são necessárias. Dados já publicados para muitos produtos alimentícios não são adequados, na maioria das vezes, para os produtos nacionais, conforme Moura et al (2003).

O objetivo deste trabalho foi aplicar uma adaptação do método desenvolvido por Hwang e Hayakawa (1979) para determinar o calor específico do suco de laranja, utilizando-se uma garrafa térmica adquirida no comércio local, adaptada para servir como calorímetro, preenchida com um líquido inerte. $\mathrm{O}$ cálculo é feito através de um balanço global de energia em um sistema considerado isolado termicamente.

\section{MATERIAIS E MÉTODOS}

\subsection{Materiais}

O calor específico do suco de laranja foi determinado experimentalmente através de uma adaptação do método proposto por Hwang e Hayakawa (1979). Um calorímetro foi construído através da modificação de uma garrafa térmica, marca INVICTA, de capacidade volumétrica de 1 litro. Uma tampa foi construída de tecnil, usinada para se adaptar à parte superior da garrafa, com abertura para colocação de um termopar. Na parte inferior da garrafa, um furo apropriado foi feito para inserir um segundo termopar. O calorímetro usado para medição do calor específico é apresentado esquematicamente na Figura 1.

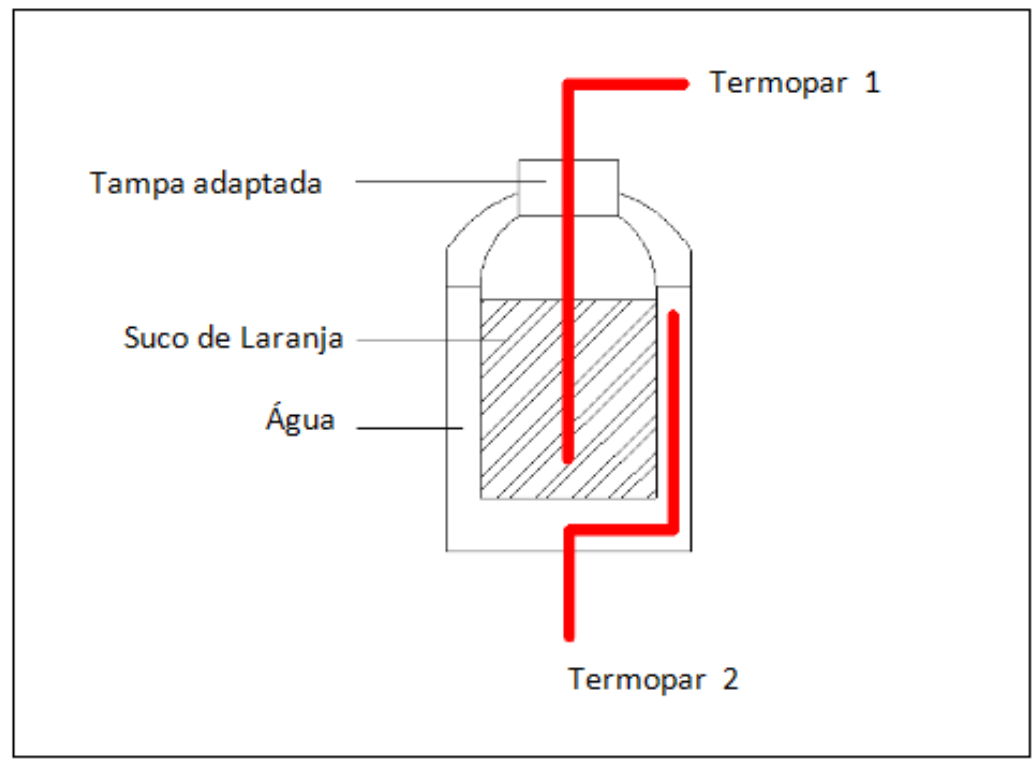

Figura 1 - Esquema do calorímetro utilizado para medição do calor específico. 
Uma massa de água de 418,0 g foi colocada na parte externa da garrafa, no espaço entre a garrafa e o corpo de plástico, de modo que o nível de água foi até cerca de 2,0 cm abaixo da borda da tampa. O sistema foi apropriadamente vedado para evitar fugas de água. A temperatura dessa massa de água foi medida pelo termopar 2 da Figura 1.

No interior da garrafa térmica foi inserido o suco de laranja, preparado a partir de seis laranjas adquiridas no mercado local, sem adição de água, com massa total de 510,0 g. A temperatura do suco de laranja foi medida pelo termopar 1 da Figura 1.

\subsection{Determinação da capacidade calorífica do calorímetro}

A capacidade térmica do calorímetro foi necessária como um fator de correção para a determinação do calor específico do suco de laranja. Um procedimento foi então desenvolvido para essa determinação.

A massa de água, inicialmente a $60^{\circ} \mathrm{C}$, foi colocada no espaço entre a garrafa de vidro e o corpo plástico da garrafa térmica, adaptada como calorímetro. As temperaturas da água e do calorímetro foram registradas continuamente por cerca de uma hora. Foi observado que, após aproximadamente 30 minutos, os conteúdos atingiram uma taxa de calor com o meio ambiente constante.

A partir da lei da conservação de energia, o calor total da água e do calorímetro, no estado inicial, deverá ser igual ao calor no estado final, após o equilíbrio, mais a taxa de calor que é perdida para a vizinhança. Adotando então o índice o para representar o estado inicial e o índice e para o estado final, quando o sistema atingiu o equilíbrio, tem-se a Equação 1:

$$
C p_{a} m_{a} T o_{a}+C p_{c} m_{c} T o_{c}=C p_{a} m_{a} T e+C p_{c} m_{c} T e-R
$$

O calor perdido para a vizinhança é considerado constante durante o experimento e pode ser determinado pela equação 2:

$$
R=\frac{d q}{d t}=\left[m_{c} C p_{c}+m_{a} C p_{a}\right]\left(\frac{d T}{d t}\right) t_{e}
$$

Substituindo a Equação 2 na Equação 1, tem-se uma expressão apropriada para a determinação 
do calor específico do calorímetro, que é a Equação 3:

$$
m_{c} C p_{c}=\frac{m_{a} C p_{a}\left[T o_{a}-T f_{a}+\left(\frac{d T}{d t}\right) t_{e}\right]}{\left[T f_{c}-T o_{c}-\left(\frac{d T}{d t}\right) t_{e}\right]}
$$

Na Equação 3 o termo $\left(\frac{d T}{d t}\right)$ representa a inclinação da curva da temperatura em função do tempo, apresentada na Figura 2, com unidade $\left({ }^{\circ} \mathrm{C} / \mathrm{s}\right)$.

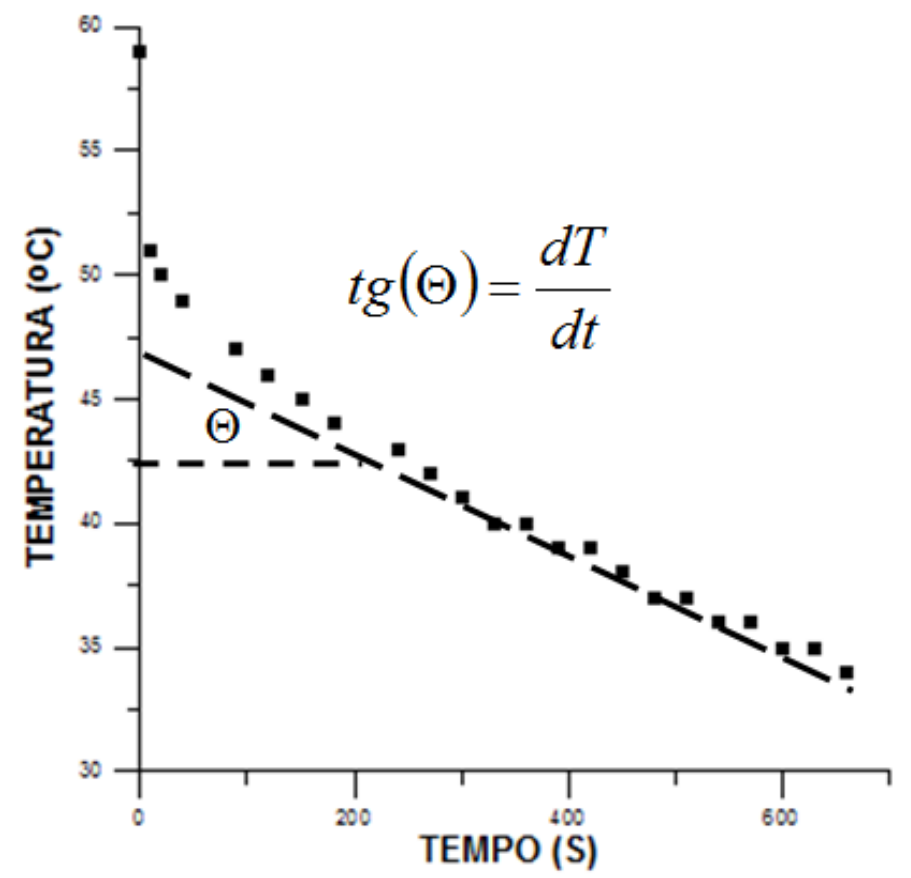

Figura 1 - Variação da temperatura da água durante o experimento com o calorímetro. 


\subsection{Determinação da Capacidade Calorífica do Suco}

O experimento é novamente realizado, agora usando o suco de laranja no interior do calorímetro. As temperaturas do suco de laranja, da água e do calorímetro são novamente monitoradas, até que se atinja o equilíbrio térmico com o meio ambiente.

Usando novamente a lei da conservação da energia, a quantidade de calor total do sistema no estado inicial deverá ser igual ao conteúdo de energia no estado final menos a parcela que é perdida para o ambiente, que dessa vez será dada pela Equação 4:

$$
R=\left[C p_{a} m_{a}+C p_{c} m_{c}+C p_{S} m_{S}\left(\frac{d T}{d t}\right) t_{e}\right.
$$

O termo $\left(\frac{d T}{d t}\right)$ da Equação 4 é obtido pelo gráfico da variação da temperatura da água com o tempo, conforme mostra a Figura 3.

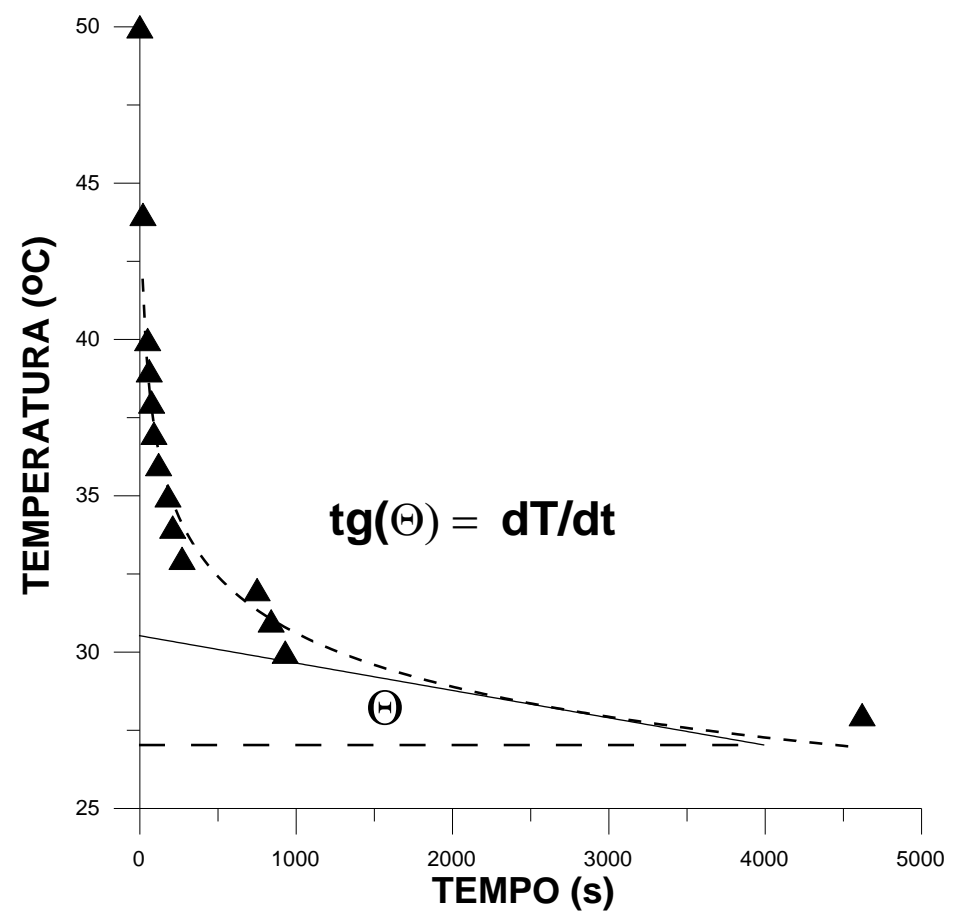

Figura 3 - Variação da temperatura da água com o tempo. 
Novamente, o balanço de energia total, agora para o processo envolvendo o suco de laranja, resulta na Equação 5:

$$
C p_{S} m_{S}=\frac{C p_{a} m_{a}\left[T f_{a}-T o_{a}-\left(\frac{d T}{d t}\right) t_{f}\right]-C p_{C} m_{C}\left[\left(\frac{d T}{d t}\right) t_{f}+T o_{C}-T f_{C}\right]}{\left[T o_{S}-T f_{S}+\left(\frac{d T}{d t}\right) t_{f}\right]}
$$

A Equação 5 representa a expressão matemática que fornece o calor específico do suco de laranja.

\section{RESULTADOS}

Após a realização dos experimentos, e considerando o resultado apresentado na Figura 1, inicialmente a Equação 3 foi utilizada para a determinação do calor específico do calorímetro utilizado, com uma inclinação encontrada de $\frac{d T}{d t}=0,0194{ }^{\circ} \mathrm{C} / \mathrm{s}$ na Figura 1 , o que resultou no calor específico do calorímetro de $C p_{C}=526,42 \mathrm{~J} / \mathrm{kg}^{\circ} \mathrm{C}$.

Na Figura 2 a inclinação encontrada foi de $\frac{d T}{d t}=0,00233{ }^{\circ} \mathrm{C} / \mathrm{s}$, o que resulta, substituído na Equação 5, no calor específico do suco de laranja de $C p_{S}=3494,85 \mathrm{~J} / \mathrm{kg}^{\circ} C$.

Para comparação do valor experimental obtido através do método, o modelo proposto por Siebel (1982), apropriado para o suco de laranja foi utilizado, conforme a Equação 6:

$$
C p=1,424 X_{H}+1,549 X_{P}+1,675 X_{F}+0,837 X_{A}+4,187 X_{W}
$$

Nessa equação, uma composição da laranja analisada em laboratório fornece os seguintes valores para os termos, conforme apresenta a Tabela 1: 
Tabela 1 - Valores apropriados das frações usadas na Equação 6

\begin{tabular}{|c|c|c|c|c|}
\hline Carboidratos $\left(\mathrm{X}_{\mathrm{H}}\right)$ & Proteína $\left(\mathrm{X}_{\mathrm{P}}\right)$ & Gordura $\left(\mathrm{X}_{\mathrm{G}}\right)$ & Cinzas $\left(\mathrm{X}_{\mathrm{C}}\right)$ & Umidade $\left(\mathrm{X}_{\mathrm{U}}\right)$ \\
\hline 0,111 & 0,008 & 0,002 & 0,004 & 0,875 \\
\hline
\end{tabular}

O resultado obtido para o calor específico do suco de laranja, através do uso da Equação 6, é igual a $C p_{S}=3,840 \mathrm{~J} / \mathrm{kg}^{\circ} \mathrm{C}$. Sendo assim, o valor predito pela literatura é bem próximo ao resultado experimental encontrado neste trabalho, apresentando um erro da ordem de $9,88 \%$.

\section{CONCLUSÃO}

O resultado experimental para o calor específico do suco de laranja, obtido através de uma metodologia de simples execução em laboratório, aproximou-se do valor predito por correlações recomendadas pela literatura. O erro encontrado, inferior a 10\%, é bastante razoável para aplicações de engenharia e pode ser atribuído a possíveis descuidos na manipulação dos dispositivos para medição de temperatura e tempo. Embora todos os pontos experimentais sejam médias de repetições, melhorias na metodologia podem ainda ser realizadas em trabalhos futuros. A metodologia pode, então, ser usada para determinações de propriedades térmicas de outros sucos de frutas, cujos valores são ainda desconhecidos, permitindo o desenvolvimento de processos industriais onde tais propriedades são necessárias ao dimensionamento de equipamentos.

\section{NOMENCLATURA}

Cp Calor específico: $\mathrm{J} / \mathrm{kg}^{\circ} \mathrm{C}$

$\mathrm{dq} / \mathrm{dt} \quad$ Taxa de calor perdido: $\mathrm{W} / \mathrm{m} . \mathrm{s}^{\circ} \mathrm{C}$

dT/dt Taxa de variação da temperatura: ${ }^{\circ} \mathrm{C} / \mathrm{s}$

$\mathrm{m} \quad$ Massa da substância: $\mathrm{kg}$

R Termo de correção para perda ou ganho de energia durante o experimento

$\mathrm{T}$ Temperatura: ${ }^{\circ} \mathrm{C}$

To Temperatura inicial: ${ }^{\circ} \mathrm{C}$

Tf Temperatura final: ${ }^{\circ} \mathrm{C}$

t Tempo: $\mathrm{s}$

$\Theta \quad$ Ângulo representando a inclinação da curva experimental

Subscrito
A Cinzas
a Água
c Calorímetro
e: Equilíbrio 


$\begin{array}{ll}\text { F } & \text { Gordura } \\ \text { f } & \text { Estado final } \\ \text { H } & \text { Carboidrato } \\ \text { P } & \text { Proteína } \\ \text { S } & \text { Suco } \\ \text { W } & \text { Umidade }\end{array}$

\section{REFERÊNCIAS}

GEORGE, R. M. A Literature Survey of Thermal Diffusivity of Food Products, Technical Bulletin, v. 73, n. 2. P. 1-23, 1990.

HWANG, M. P.; HAYAKAWA, K. A Specific Heat Calorimeter for Foods, Journal of Food Science, v. 44, n.2, p. 435-448, 1979.

MOURA et al. Propriedades Termofísicas de Soluções Modelo Similares a Sucos-Parte I, Ciênc. Tecnol. Aliment., 23(1): 62-68, jan-abr, 2003.

POLLEY, S. L. et al. A Compilation of Thermal Properties of Foods, Food Technology, v. 34, n. 11, p. 76-94, 1980.

SIEBEL, J. E. Specific Heat of Various Products, Ice Refrig., v. 2, p. 256-257, 1982.

TELIS-ROMERO et al. Thermophysical Properties of Brazilian Orange Juice as Affected by Temperature and Water Content, Journal of Food Engineering, v. 38, p. 27-40, 1998.

Agradecimento: Os autores agradecem o apoio financeiro provido pela FAPEMIG (PCE - 00201-14: Participação Coletiva em Eventos Técnicos-Científicos). 